
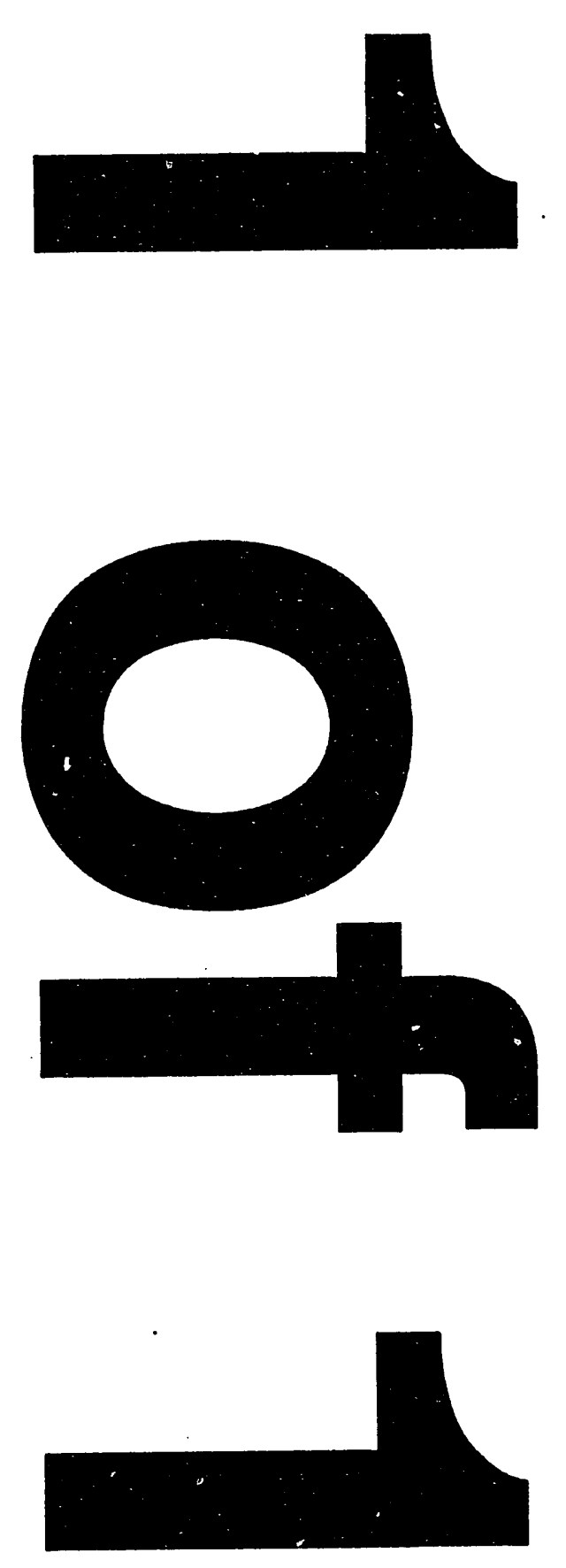
LBL-34841

\title{
Detection Geometry and Reconstruction Error in Magnetic Source Imaging
}

\author{
Paul Hughett and Thomas F. Budinger
}

Life Sciences Division

Lawrence Berkeley Laboratory

and

Department of Electrical Engineering and Computer Sciences

University of California

Berkeley, CA 94720

November 1993

This work was supported in part by a gift from the Advanced Technology Systems Division of the General Electric Company and in part by the Direcior, Office of Energy Research, Office of Health and Environmental Research, Medical Applications and Biophysical Research Division of the Department of Energy under Contract No. DE-AC03-76SFO0098.

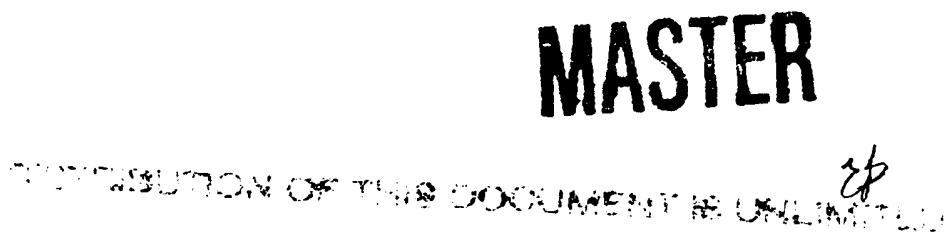




\title{
Detection Geometry and Reconstruction Error in Magnetic Source Imaging
}

\author{
Paul Hughett and Thomas F. Budinger
}

\begin{abstract}
A recentiy developed reconstruction algorithm for magnetic source imaging exploits prior knowledge about source location, source power density, detector geometry, and detector noise power to obtain an explicit estimate of the reconstruction error.

This paper demonstrates the application of the new algorithm to the optimal design of practical detector arrays to minimize the reconstruction error in specific applications. For a representative configuration for magnetocardiography, the optimal array width (for minimum reconstruction error) varies from 19 to $28 \mathrm{~cm}$ depending on the assumed source depth, number of detectors, source power, and noise power. The reconstruction accuracy ranges from $5 \%$ of the a priori standard deviation for the sources nearest the detector plane to $95 \%$ of the a priori deviation for the deepest sources. The reconstruction error was found to depend on accidental alignments between dipole sources and point detectors, indicating that a more sophisticated model is required for accurate estimates of reconstruction error. The error calculation is fast, taking about a second for this problem on a workstation-class computer.

The availability of a method for rapidly computing the reconstruction error for any given source characteristics and detector geometry will facilitate the optimal design of magnetometer array size, element spacing, and orientation for specific applications in biomagnetic and geomagnetic source imaging.
\end{abstract}

Keywords- Magnetic source imaging; magnetoencephalography; inverse problems.

\section{INTRODUCTION}

Magnetic source imaging is the reconstruction of the current distribution inside some inaccessible volume from magnetic field measurements made outside the volume. Prior information as to the expected signal power of each source and expected noise power of each detector can be used to constrain the reconstruction and improve its accuracy.

The optimal constrained linear inverse method (OCLIM) [1] is a new reconstruction algorithm for magnetic source imaging that uses such prior information to minimize the mean-square reconstruction error. OCLIM generalizei the minimum-norm least-squares $[2 ; 3]$ and weighted pseudoinverse[4] methods by incorporating non-uniform and correlated priors for both signal and noise.

OCLIM also provides explicit estimates of the (total) reconstruction error and the standard error for each reconstructed source amplitude. The purpose of this paper is

This work was supported in part by a gift from the Advanced Technology Systems Division of the General Electric Company and in part by the Director, Office of Energy Reseanch, Office of Health and Environmental Research, Medical Applications and Biophysical Research Division of the Department of Energy under Contract No. DE-AC03-76SF00098.

Paul Hughett and Thomas F. Budinger are with the Life Sciences Division, Lawrence Berkeley Laboratory and the Department of Electrical Engineering and Computer Sciences, 207-91 Cory Hall, Univers:ty of Califomia at Berkeley, Berkeley CA 94720. to illustrate how these error estimates can be used in the design of magnetometer arrays.

\section{Optimal Constrained linear Inverse Method}

OCLIM assumes that the unknown current distribution $\ddot{J}(\vec{r})$ can be written as a weighted sum of $N$ known elementary sources $\vec{J}_{n}(\vec{r})$ to obtain

$$
\vec{J}(\vec{r})=\sum_{n=1}^{N} q_{n} \vec{J}_{n}(\vec{r})
$$

Each elementary source $\vec{J}_{n}(\vec{r})$ is a vector-valued function giving the vector current density at any position $\vec{r}$. The source positions are fixed a priori; only the source amplitudes $q_{n}$ are unknown and must be estimated.

Then the forward problem can be written in the form

$$
\mathbf{b}=\mathbf{F q}+\mathbf{w}
$$

where $\mathrm{q}$ is the vector of source amplitudes, $\mathrm{b}$ is a vector of field measurements, $w$ is a vector of noise amplitudes, and $\mathbf{F}$ is the forward transfer matrix.

The vector $q$ of source amplitudes $q_{n}$ is assumed to be a random vector with mean zero and covariance matrix $\mathrm{A}=\mathrm{E} \mathrm{qq}^{T}$ with entries $\alpha_{i j}^{2}=\mathrm{E} q_{i} q_{j}$.

The noise vector $w$ is assumed to be a random vector with mean zero and covariance matrix $\Sigma=E$ ww $w^{T}$ with entries $\sigma_{i j}^{2}=E w_{i} w_{j}$. Each diagonal entry $\sigma_{m m}^{2}$ is the expected noise power of the $m$ th detector.

Nuw suppose that each source $\vec{J}_{n}$ is a current dipole with moment $\vec{q}_{n}$ at position $\vec{p}_{n}$ and that the mth detector measures the ccmponent of the field in direction $\vec{s}_{m}$ at position $\vec{r}_{, \text {n }}$. Ther the forward transfer matrix $\mathbf{F}$ has entries

$$
F_{m n}=\frac{\mu_{0}}{4 \pi} \cdot \frac{\vec{s}_{m} \cdot \vec{q}_{n} \times\left(\vec{r}_{m}-\vec{p}_{n}\right)}{\left\|\vec{r}_{m}-\vec{p}_{r}\right\|^{3}} .
$$

The OCLIM estimate of the unknown source amplitudes takes the form $\hat{\mathbf{q}}=\mathbf{H b}$ where $\mathbf{H}$ is chosen to minimize the mean square error $\eta^{2}=E\|q-\hat{q}\|^{2}$. The value of $H$ is

$$
\mathbf{H}=\mathbf{A F ^ { T }}\left(\mathbf{F A F ^ { T } + \Sigma ) ^ { - 1 }}\right.
$$

Given that $q$ and $\mathbf{w}$ are jointly Gaussian, the a posteriori covariance is

$$
\hat{\mathbf{A}}=\mathbf{A}-\mathbf{A F}^{T}\left(\mathbf{F A} \mathbf{F}^{T}+\boldsymbol{\Sigma}\right)^{-1} \mathbf{F A}
$$


The diagonal elements of $\hat{\mathbf{A}}$ are denoted by $\hat{\alpha}_{n n}^{2}$; each is the variance of the corresponding reconstructed source amplitude $\hat{q}_{n}$; and $\hat{\alpha}_{n n}$ is that standaru error of the reconstructed source amplitude $\hat{q}_{n}$. The total reconstruction error is

$$
\eta^{2}=\operatorname{Tr}(\hat{\mathbf{A}})=\sum_{n} \hat{\alpha}_{n n}^{2}
$$

and is the quantity minimized.

\section{A Tool for Magnetometer Array Design}

The total error $\eta^{2}$ and the individual source variances $\hat{\alpha}_{n n}^{2}$ provide a new tool for magnetometer array design and have the following useful properties.

The reconstruction error depends only on the geometrical configuration and the noise and source priors; it does not depend on the specific field measurements. The reconstruction error for a particular configuration can be computed in a few seconds on a workstation-class computer. This is much faster than Monte Carlo methods.

Arbitrary source and detector positions are allowed. Correlated noise can be used; this allows modelling the effects of external magnetic interference.

The method should generalize to distributed (non-dipole) current sources and arbitrary detector coil shapes.

The minimum-norm least-squares (MNLS) [2; 3] and Shim-Cho weighted pseudoinverse [4] methods are included as special cases by the apppropriate choice of priors.

\section{An Example Source/Detector Configuration}

The remainder of this paper is devoted to an analysis of the example source and magnetometer configuration shown in figure 1. The source volume is $12 \times 12 \times 12 \mathrm{~cm}^{3}$ and contains a $4 \times 4 \times 4$ cubical grid of horizontal dipole pairs; all sources have the same expected signal power $\alpha^{2}=1(\mu \mathrm{A}-\mathrm{m})^{2}$. Each horizontal plane in the source volume contains a $4 \times 4$ array of in-plane dipole pairs as shown in figure 2; the use of two orthogonal dipoles allows the representation of a dipole with arbitrary orientation and magnitude within the plane. The detector plane contains a $12 \times 12$ grid of detectors sampling the vertical component of the field; all detectors have the same expected noise power $\sigma^{2}=9 \times 10^{-26} \mathrm{fT}$. The nominal array width is $23 \mathrm{~cm}$; the nominal source depth, or distance from the detector array to the top of the source volume, is $2 \mathrm{~cm}$.

The following sections will explore the effects of varying the array width, the source depth, and the number of detectors used.

\section{v. ERror vs ARray WIDTH AND Source DePTH}

Figure 3 shows how the total relative reconstruction error $\eta^{2} / \alpha^{2}$ varies as a function of the array width and the source depth. For every source depth considered, there is an optimal array width and that width increases as the source depth increases. The particular optimal widths found here apply only to the particular number of detectors, source variance, and noise variance used to compute them.

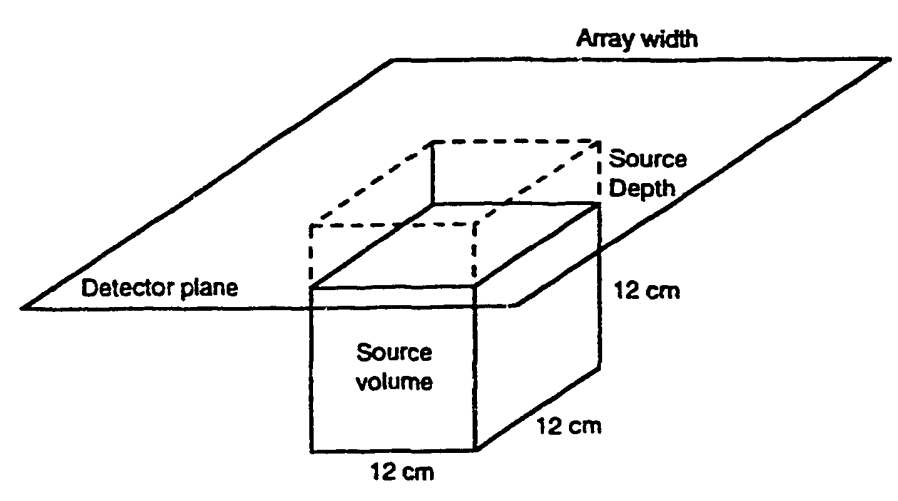

Fig. 1. Example source and detector configuration.

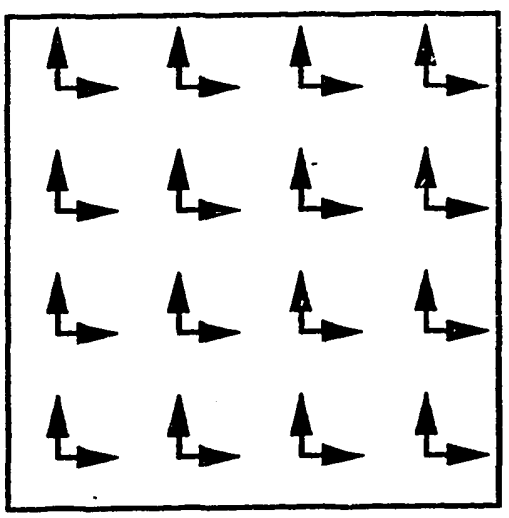

Fig. 2. Typical horizontal plane of sources in the source volume.

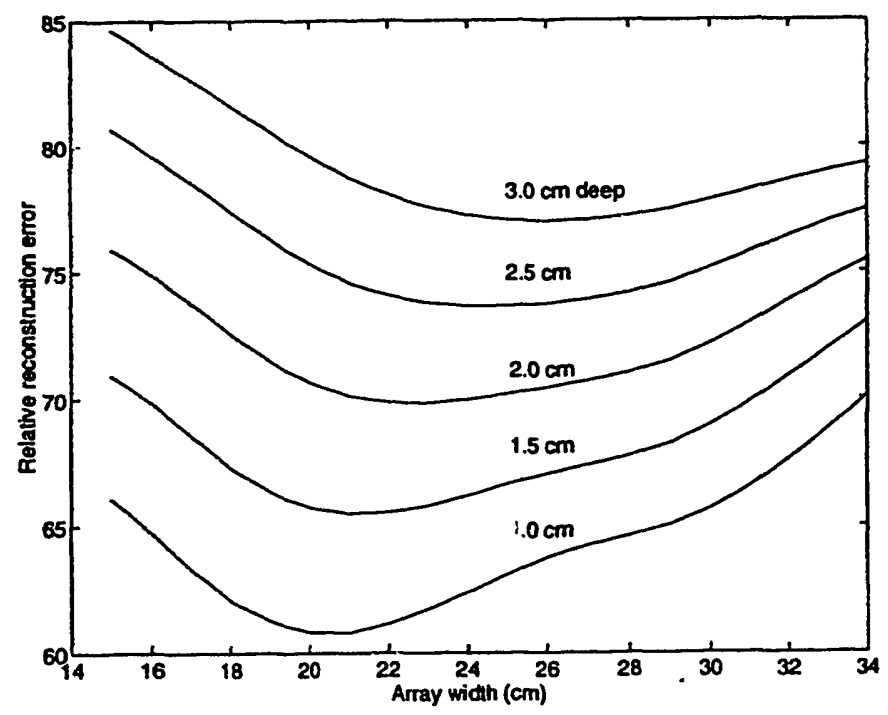

Fig. 3. Total reconstruction error versus array width and source depth 


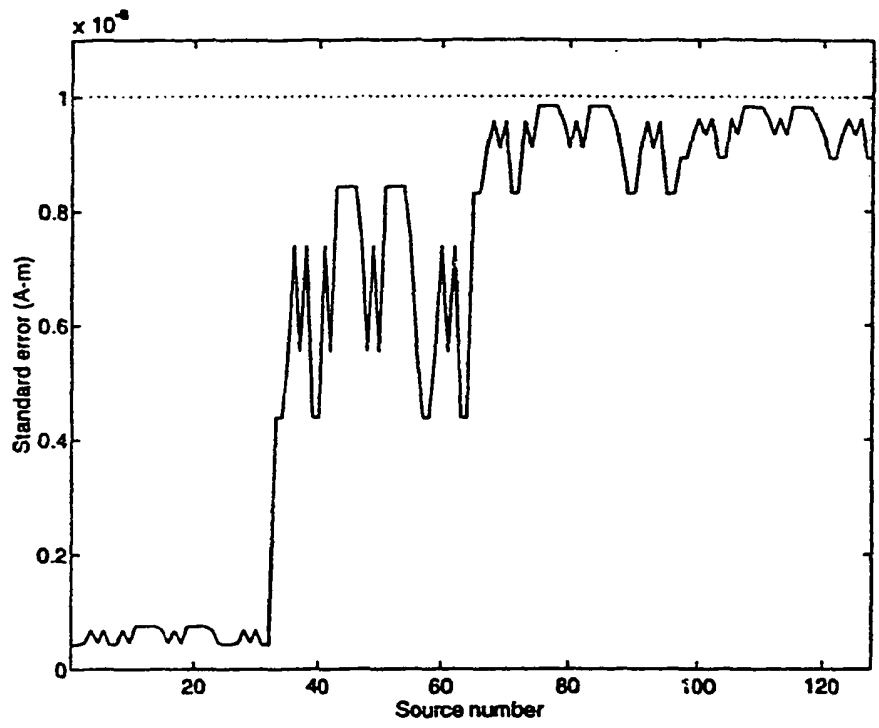

Fig. 4. Standard error for each source using the nominal detector configuration and priors.

\section{STANDARD ERror for Each Source}

Figure 4 shows the standard error $\hat{\alpha}_{n n}$ for each source, using the nominal values for array width, source depth, and number of detectors. The a priori standard deviation $\alpha_{n n}$ is shown as the dotted line across the top of the figure. The standard errors for sources 1-32, all in the topmost plane of the source volume, are about $5 \%$ of their a priori values. That is, this detector configuration yields reconstruction errors for these sources that are about $5 \%$ of their initial uncertainties. Detectors 33-64 are in the second plane down; their uncertainties are reduced to $40-80 \%$ of their initial values. Sources 65-128 are in the bottom two planes; their uncertainties are hardly reduced from their initial values. That is, this detector array (for the assumed priors) gives good reconstructions for the topmost plarie of sources, mediocre results for the second plane, and poor results for the bottom two planes.

\section{ERror vS WidTh AND NUMBER of Detectors}

Figure 5 shows the total relative reconstruction error versus array width for five different numbers of detectors ranging from an $8 \times 8$ square grid to a $16 \times 16$ square grid. There is an optimal array width for each size of grid; that width increases as the number of detectors increases.

Note, however, that both the $8 \times 8$ and $10 \times 10$ curves display a bimodal characteristic. This is due to the accidental alignment of source and detector positions as shown in the plan views of figure 6 . The plus signs mark detector positions; the open circles mark source positions. The lefthand plot corresponds to the minimum error (at width 19 $\mathrm{cm}$ ) for an $8 \times 8$ array; the sources generally fall between the detectors. The righthand plot corresponds to the local maximum at $23 \mathrm{~cm}$; the sources fall almost directly under the detector positions.

Since dipole sources are used only for convenience in approximating a continuous unknown current distribution,

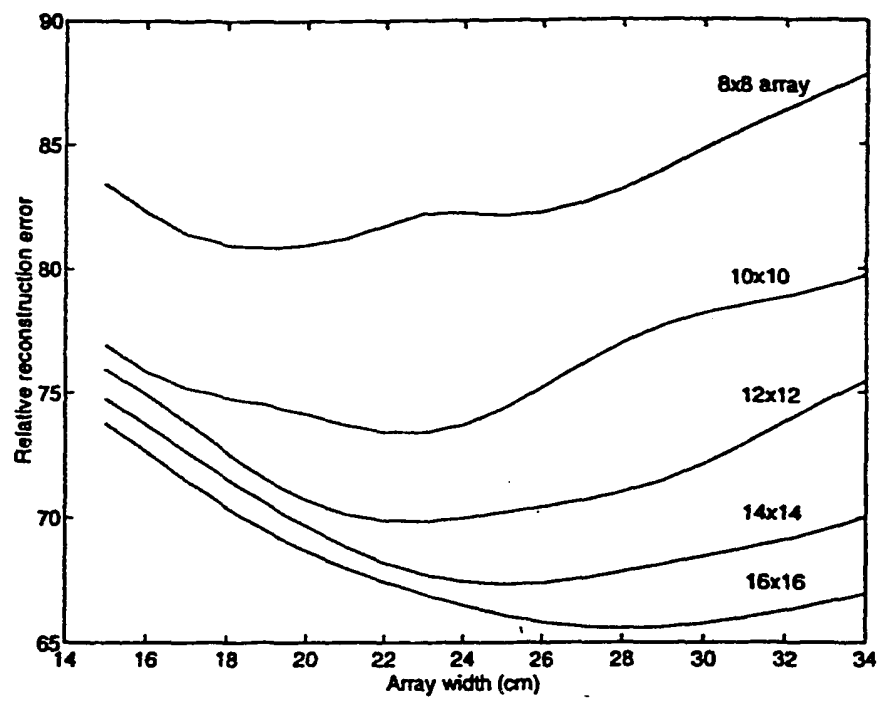

Fig. 5. Total reconstruction error versus array width and number of detectors.

this variation in reconstruction error must be considered an artifact of the simple dipole source model. The use of point detectors also contributes to the problem. More work is needed to create source and detector models that are immune to these accidental variations.

Figure 7 shows the optimal $8 \times 8$ and $16 \times 16$ arrays. The optimal width increases as the number of detectors increases but less than proportionally to the number of detectors per side; the optimal detector speacing decreases.

\section{CoNClUSions}

The reconstruction error is potentially useful as a quality metric for magnetometer array design.

Accidental alignments between dipole current sources and point detectors can distort the computed reconstruction error.

The optimal width increases as the array-to-source distance increases. The optimal width of a planar magnetometer array is subproportional to the number of detectors used per side.

\section{REFERENCES}

[1] Paul Hughett. An optimal constrained linear inverse method for magnetic source imaging. Technical Report LBL 34512 , Lawrence Berkeley Laboratory, September 1993.

[2] Jia-Zhu Wang, Samuel J. Williamson, and Lloyd Kaufman. Magnetic source images determined by a lead-field analysis: The unique minimum-norm least-squares estimation. IEEE Trans. Biomed. Eng., BME-39(7):665-675, July 1992.

[3] Jia-Zhu Wang. Minimum-norm least-squares estimation: Magnetic source images for a spherical model head. IEEE Trans. Biomed. Eng., BME-40(4):387-396, April 1993.

[4] Y.S. Shim and Z. H. Cho. SVD pseudoinversion image reconstruction. IEEE Trans. ASSP, ASSP-29(4):904-909, August 1981. 

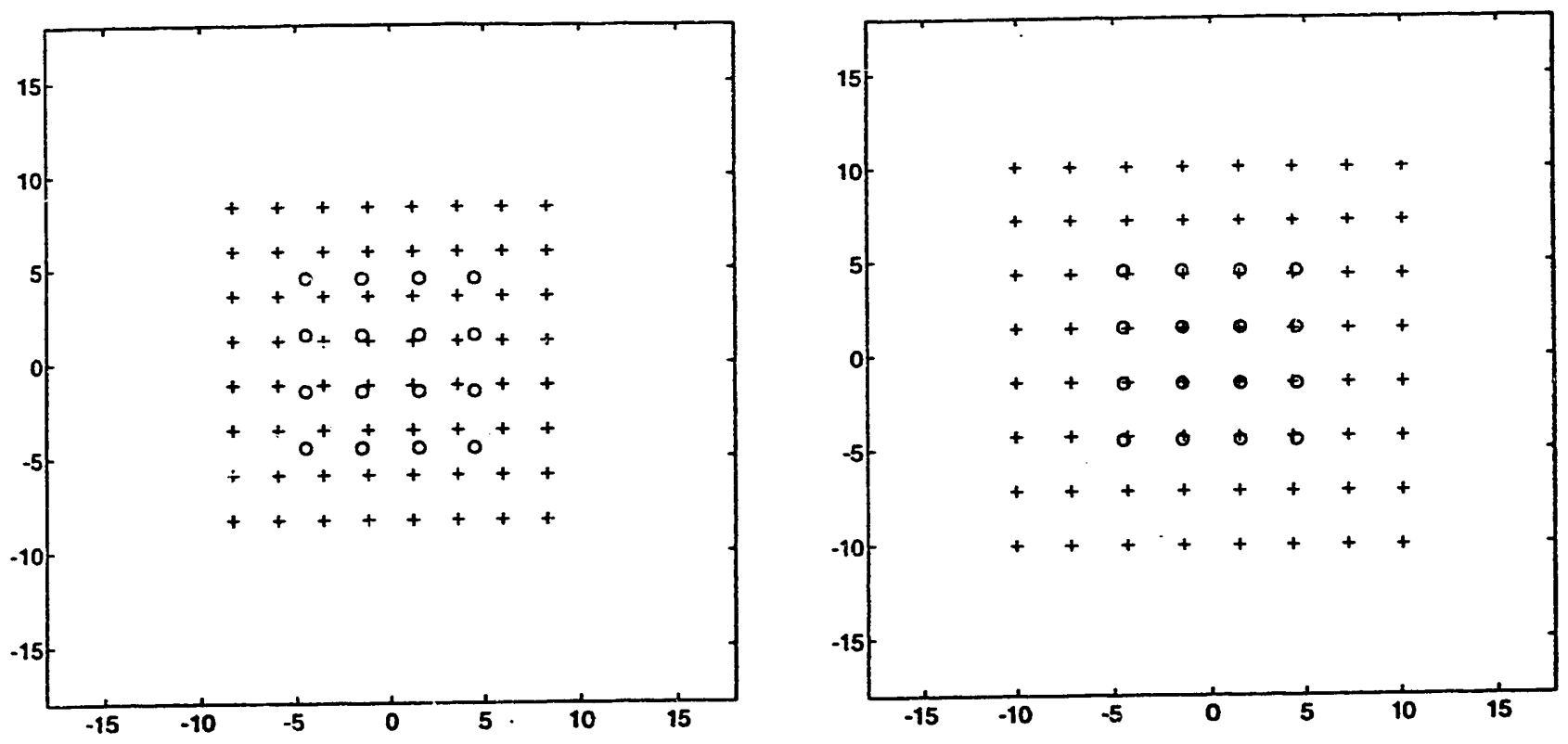

Fig. 6. Source and detector aligmments. The lefthand plot shows the source $(0)$ and detector $(t)$ locations in plan view for the minimum-error Fig. 6. Source and detector alignments. The lefthand plot shows the source (o) and detector (t) configuration with local maximum error. The sources fall directly below the detector locations.
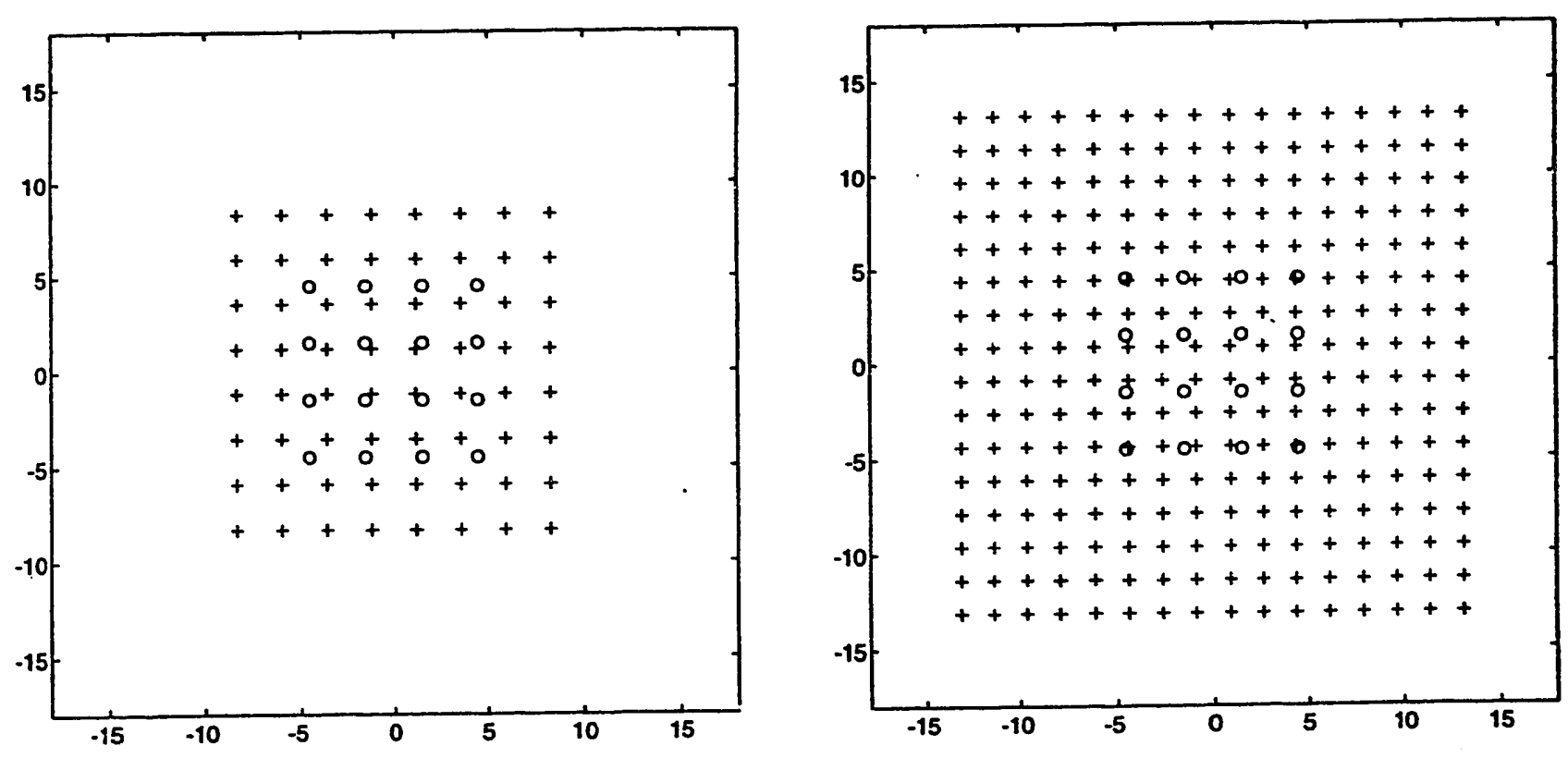

Fig. 7. Optimal array width versus number of detectors. The lefthand plot shows the optimal $8 \times 8$ array; the righthand plot the optimal $16 \times 16$ Fig. 7. Optimal width increases less than proportionally to the number of detectors per side and the optimal detector spacing decreases. 


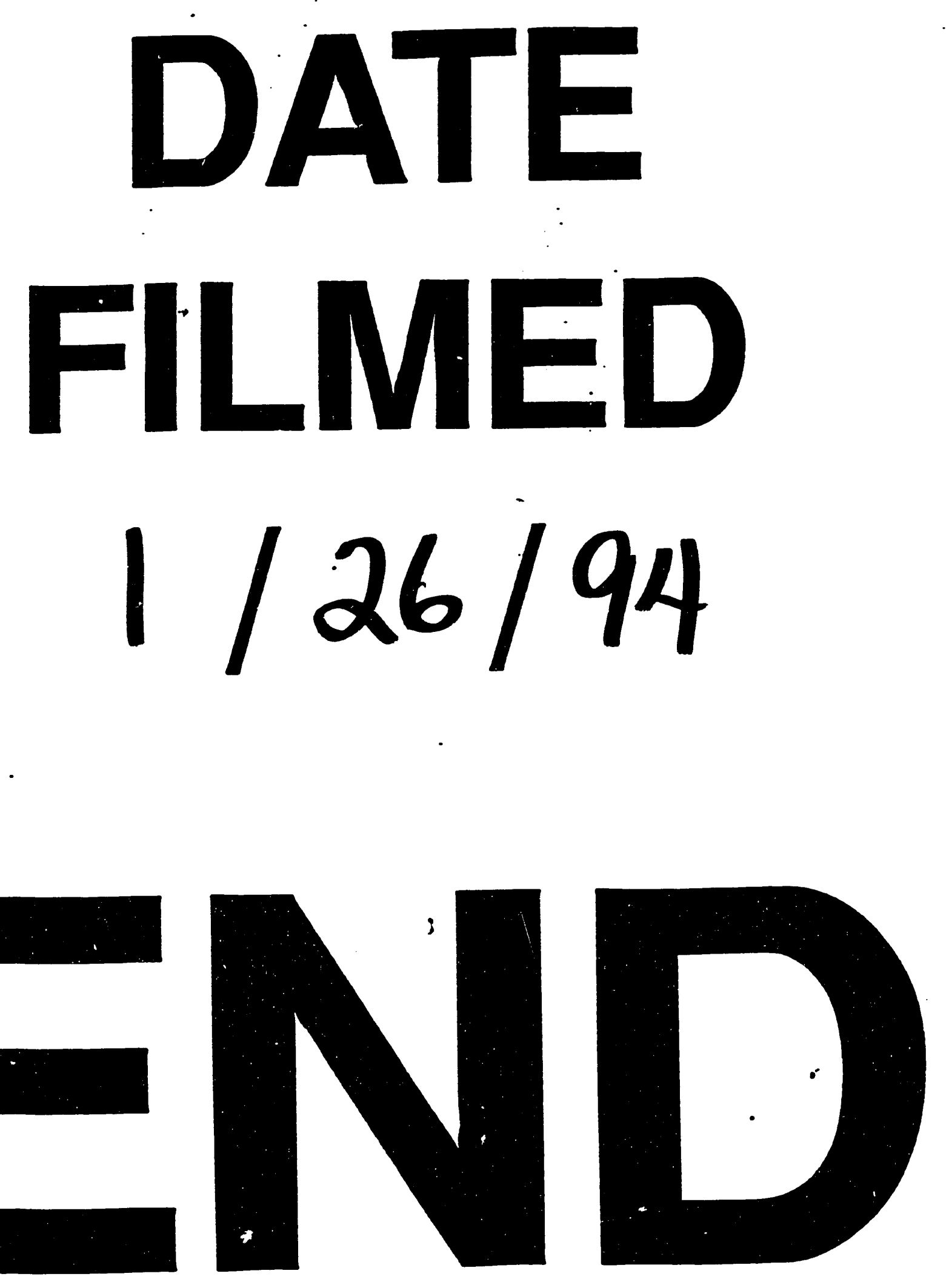

